\title{
A Novel Universal Reducer Integrating a Planetary Gear Mechanism with an RCRCR Spatial Mechanism
}

\author{
Cheng Gu ${ }^{1,2}-$ Xinbo Chen ${ }^{1,2, *}$ \\ 1 Tongji University, Clean Energy Automotive Engineering Center, China \\ 2 Tongji University, School of Automotive Studies, China
}

\begin{abstract}
A novel speed reducer with self-adaptability to variable transmission angles is proposed in this paper, in which a planetary gear mechanism with a difference of a few teeth and an RCRCR (R-revolute pair, C-cylindrical pair) spatial mechanism are integrated. The difference of a few teeth presents the advantages of high transmission ratio and compact structure, while the RCRCR spatial mechanism achieves the translational motion of the planetary gear in space. Theoretical kinematics analyses are carried out to prove the motion of each component in space. Models are established in the Automatic Dynamic Analysis of Mechanical Systems (ADAMS) to verify the performances of universal transmission and torque fluctuation tolerance. The simulation results demonstrate that the proposed speed reducer maintains a stable transmission ratio with variable transmission angles, presenting a high level of tolerance with the position perturbations. Strength analysis and manufacturing and lubrication guidelines of the prototype design are presented.

Keywords: integrate, self-adaptability, planetary gear mechanism, few teeth difference, RCRCR spatial mechanism, universal reducer

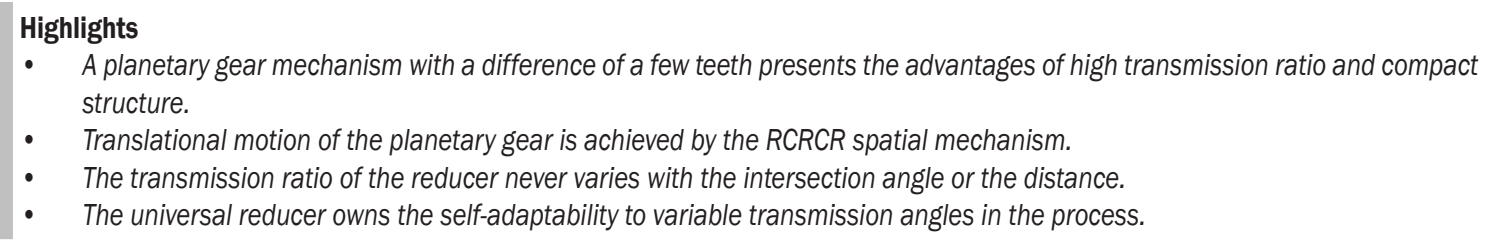

\section{INTRODUCTION}

Speed reducers, which perform the function of speed and torque conversion, are widely utilized in modern machine applications. A common speed reducer can offer speed reduction with fixed relative position between the input shaft and output shaft. For instance, to reduce the speed between parallel shafts of bicycles, $\mathrm{Wu}$ and Chen proposed an eight-speed internal cylinder gear hub [1]. Instead of traditional four discs, Blagojevic et al. adopted two cycloid discs to achieve two-stage speed reduction with the advantages of compact structure, good load distribution and dynamic balance [2]. Furthermore, it is proved that efficiency is decreased with the increase of friction and contact forces [3]. By numerical simulation and experiment verification, Concli and Gorla studied the influences of parameters on the planetary speed reducer, including lubricant temperature and rotational speed [4]. Speed reduction transmission with fixed intersect shafts can be achieved with bevel gear mechanisms. Wang summarized the mathematical models of the spiral bevel gear, including the applications of thermal analysis, frictional analysis and advanced manufacturing technology [5]. For spiral bevels and hypoid gears manufactured with the duplex helical method, Zhang et al. calculated the basic machinetool settings with a new method [6]. Worm reducers or the screw gear reducer transmits two screwed vertical shafts. Dudás established the model of a new worm gear drive having point-like contact and conducted simulations to verify the better tolerance with misalignment [7]. For the case of a non-vertical intersection angle between the shafts, by defining and calculating the sliding rate, Ding et al. proposed a parameter selection criterion of the helix curve meshing wheel (HCMW) [8]. As it is transmitted between the space curves instead of space surfaces, the load capacity is limited, and the transmission ratio is unstable. However, the reducers mentioned above have no ability to adapt to variable intersection angles in the transmission process. A couple of universal joints are usually introduced, which leads to a more complicated connection relationship among the shafts and a larger size of transmission system in the axial direction.

Our research team proposed a speed reducer of externally engaged gears with the RCRCR mechanism [9]. Litvin and Tan proposed a general approach in determining the singularities of the motion and displacement functions of the RCRCR mechanism [10]. Through several tests of the prototype, the phenomenon of "lock up" among the components appeared in the process of transmission, preventing it from rotating smoothly. After theoretical analyses and prototype tests, it shows that, except the factors 
of assembly and lubrication, uneven stress plays an important role as the torque is transmitted by only one pair of eccentric shafts. In contrast, with the same transmission ratio, the speed reducer achieved by synchronous pulleys or external gear pair occupies a larger size than the internal gear pair, which obviously limits the application of the mechanism ([11] to [13]). The "few teeth difference" planetary gear mechanism, named for the small difference between the engaged gear teeth, has been widely used in mechanisms for the outstanding advantages of compact structure, large transmission ratio and high efficiency ([14] to [16]). For instance, if the teeth of the centre gear and planetary gear are 50 and 49 respectively, the transmission ratio is 50 , while the eccentric distance is only $m / 2$, in which $m$ is the module of gear.

Regarding the two problems mentioned above, a novel planetary gear reducer of internal gearing with a difference of a few teeth is proposed in this paper to achieve universal transmission and compact structure. A common application for such a universal reducer is the transmission system in the industry, as depicted in Fig. 1. In the traditional reduction configuration of Fig. 1a, two extra couplings are introduced to transmit the power, which requires high precision alignment. However, errors and tolerances caused by manufacturing, assembly, load, friction and temperature are inevitable, which requires significant efforts in installation and maintenance. In Fig. 1b, with the fault tolerance ability to compensate the relative displacement error, even for the inconsistent axes of the transmission components, there is great convenience and feasibility in the installation and operation processes.
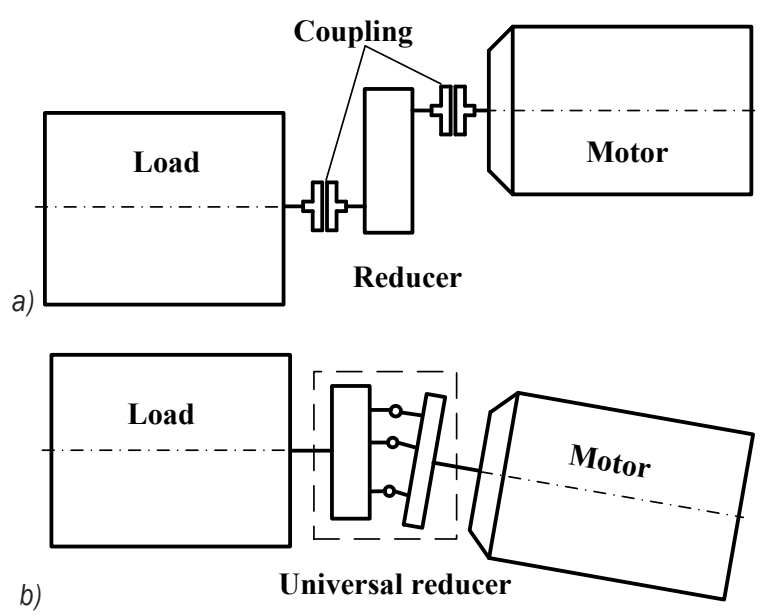

Fig. 1 Application of universal reducer; a) traditional reduction configuration with two extra couplings, and b) the reduction configuration with the universal reducer

\section{MECHANISM THEORY}

The universal reducer integrates a planetary gear mechanism of internal gearing and an RCRCR spatial mechanism; the architectures are introduced in detail below.

\subsection{Planetary Gear Mechanism}

A K-H (K-centre gear, H-planetary carrier) planetary gear is depicted in Fig. 2, which is composed of centre gear $\mathrm{O}_{1}$ with teeth $z_{1}$, planetary gear $\mathrm{O}_{2}$ with teeth $z_{2}$, and planetary carrier $\mathrm{H}$ connecting $\mathrm{O}_{1}$ and $\mathrm{O}_{2}$. According to the degree of freedom (DOF) equation, the mechanism DOF obviously is 2, which means a certain restriction is needed to guarantee a stable transmission ratio.

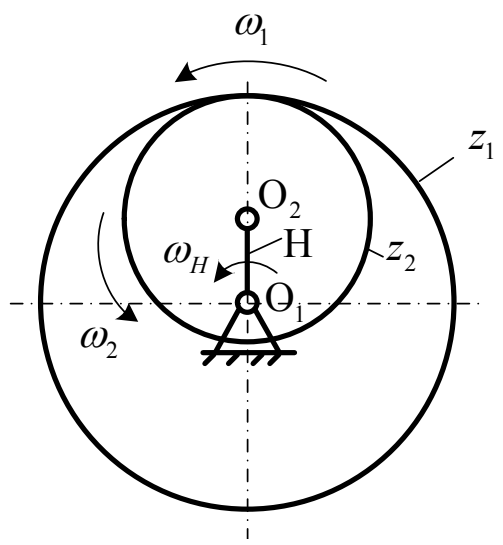

Fig. 2. Planetary gear train

Define the transmission ratio as the angular velocity ratio of the input shaft to the output shaft. Taking the planetary carrier angular velocity $\omega_{H}$ as a reference, the angular velocity transmission ratio of gear 2 to gear 1 is satisfied as Eq. (1) shows.

$$
i_{21}^{H}=\frac{\omega_{2}-\omega_{H}}{\omega_{1}-\omega_{H}}=+\frac{z_{1}}{z_{2}}
$$

where $\omega_{1}, \omega_{2}$ and $\omega_{H}$ are angular velocities of the centre gear, planetary gear and planetary carrier, respectively, $z_{1}$ and $z_{2}$ are the centre gear and planetary gear teeth respectively. The symbol "+" means that the centre gear and planetary gear rotate in the same direction (planetary carrier $\mathrm{H}$ acts as the reference object), while the symbol "_" means that the centre gear and planetary gear rotate in the opposite direction.

This needs to be emphasized for the case of $\omega_{1}=\omega_{H}$. Although, according to Eq. (1): 


$$
i_{21}^{H}=\frac{\omega_{2}-\omega_{H}}{\omega_{1}-\omega_{H}}=\frac{0}{0},
$$

as an integral, the relative positions of the centre gear, planetary gear, and planetary carrier never change in the transmission process, as depicted in Fig. 1. Consequently, the transmission ratio is 1 for certainty as $\omega_{1}=\omega_{2}=\omega_{H}$.

To obtain a stable transmission ratio, supposing planetary gear $\mathrm{O}_{2}$ is constrained to perform a translational motion in space by the RCRCR spatial mechanism proposed below, which means $\omega_{2}=0$, the following equations can be deduced according to Eq. (1).

$$
\begin{gathered}
i_{21}^{H}=\frac{-\omega_{H}}{\omega_{1}-\omega_{H}}=\frac{z_{1}}{z_{2}}, \\
\frac{\omega_{H}}{\omega_{1}}=\frac{z_{1}}{z_{1}-z_{2}} .
\end{gathered}
$$

Consequently, taking the angular velocities of planetary carrier $\omega_{H}$ and centre gear $\omega_{1}$ as the input and output velocities respectively, a stable transmission ratio is concluded. To obtain the transmission ratio in Eq. (4), it can also obviously be achieved by adopting two external gearing pairs with three gears at least, making one of the gears an idler gear. However, it would increase the mechanism components and make the transmission system more complicated.

As a comparison, if an external gearing pair is adopted, the transmission ratio of the planetary gear would be deduced as below.

$$
\frac{\omega_{H}}{\omega_{1}}=\frac{z_{1}}{z_{1}+z_{2}} .
$$

Comparing the results of Eqs. (4) and (5), it is proved that, with the same gear parameters, the transmission ratio of the mechanism with internal gearing is much larger than that with external gearing.

\subsection{RCRCR Spatial Mechanism}

To achieve the translational motion of the planetary gear, the RCRCR spatial mechanism is proposed in Fig. 3. The spatial mechanism is composed of shafts "1", "2", "3", "4" and stander " 0 ", including three revolution pairs $(\mathrm{R})$ and two cylinder pairs $(\mathrm{C})$. The characteristics of the RCRCR spatial mechanism are stated below in detail. The axes of the revolution pairs are denoted as "a", "d" and "f", in which, "a" and "d" are the fixed axes of the intersection shafts; the floating axes of the cylinder pairs are denoted as "b" and "c", which are parallel to the fixed axes "a" and
" $d$ " respectively. The intersection distance $d_{0}$ between the connecting shafts " $a$ " and " $d$ " is equal to the distance " $d_{1}$ " between the connecting shafts " $b$ " and "c". The distance $r_{1}$ between "a" and "b" is equal to the distance $r_{h}$ between "c" and "d". The intersection angle $\alpha_{14}$ between "a" and "d" equals $\alpha_{23}$ between "b" and "c".

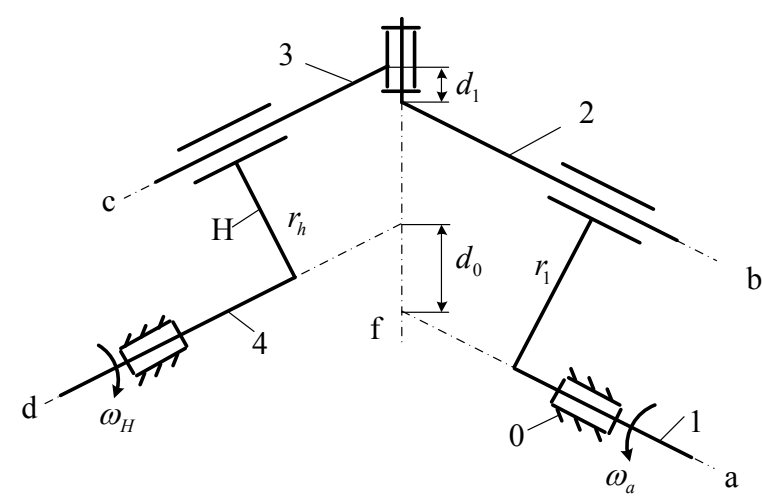

Fig. 3. RCRCR spatial mechanism

The degree of freedom (DOF) of the RCRCR spatial mechanism is calculated below.

$$
F=\sum f-\lambda=\sum_{k=1}^{\lambda-1} k p_{k}-\lambda,
$$

in which $\Sigma f$ is the DOF of the open chain mechanism of the space-connecting shaft, which is 7, supposing the mechanism is disconnected with the stander. $\lambda$ is the DOF of the end link of the open chain, which is 6 .

As a result, the RCRCR mechanism is of $1 \mathrm{DOF}$, which proves the transmission feasibility.

$$
F=\sum f-\lambda=1
$$

\section{KINEMATIC ANALYSES}

Kinematic analysis is carried out to prove the translational motion of the connecting shafts in the RCRCR spatial mechanism. For the convenience of analysis, a right-hand rectangular coordinate system is constructed on each component depicted in Fig. 4. $Z$-axis is along the axis of each kinematic pair, and $X$-axis is along the common perpendicular of two axes of the adjacent kinematic pairs. $h_{j}$ is the shortest distance between $\mathrm{z}_{i}$ and $\mathrm{z}_{j}$ along the axis $\mathrm{x}_{j} . s_{j}$ is the distance between $\mathrm{x}_{i}$ and $\mathrm{x}_{j}$ along the axis $\mathrm{z}_{i} . \alpha_{i j}$ is the angle between $\mathrm{z}_{i}$ and $\mathrm{z}_{j}$ along the axis $\mathrm{x}_{j} . \theta_{j}$ is the rotating angle of the corresponding component $i$.

A direction cosine matrix is employed to express the exchanging relationships between coordinate 
system $x_{i}\left(y_{i}\right) z_{i}$ and $x_{j}\left(y_{j}\right) z_{j}$, which can be written as the equation (8).

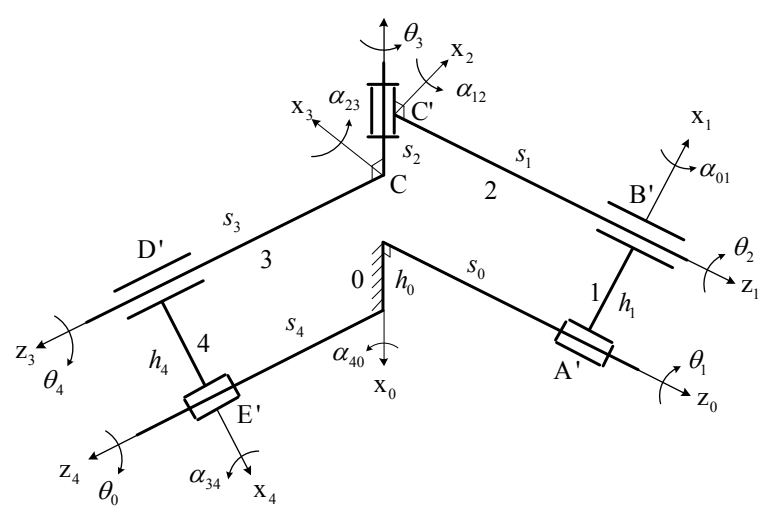

Fig. 4. RCRCR spatial mechanism

$$
\left[C_{i j}\right]=\left[\begin{array}{ccc}
\cos \theta_{j} & -\sin \theta_{j} \cos \alpha_{i j} & \sin \theta_{j} \sin \alpha_{i j} \\
\sin \theta_{j} & \cos \theta_{j} \cos \alpha_{i j} & -\cos \theta_{j} \sin \alpha_{i j} \\
0 & \sin \alpha_{i j} & \cos \alpha_{i j}
\end{array}\right] .
$$

Along axes $z_{2}$ and $z_{3}$, the closed loop can be separated into two parts: fixed chain 3-4-0-1-2 and floating chain 2-3. According to the geometric equivalence property of the kinematics, the direction cosine of the axes $z_{2}$ and $z_{3}$ in fixed chain 3-4-0-12 equals to that in floating chain 2-3, which can be defined by the following equation.

$$
\cos \left(z_{2}, z_{3}\right)_{3-4-0-1-2}=\cos \left(z_{2}, z_{3}\right)_{2-3}=\cos \alpha_{23},
$$

in which,

$$
\cos \left(z_{2}, z_{3}\right)_{3-4-0-1-2}=[0,0,1]\left[C_{34}\right]\left[C_{40}\right]\left[C_{01}\right]\left[C_{12}\right][0,01]^{T} .
$$

Through calculation, Eq. (5) can be simplified as

$$
\cos \left(\theta_{1}+\theta_{2}\right)=0 .
$$

Thus,

$$
\theta_{2}=270^{\circ}-\theta_{1} \text {. }
$$

According to the equal geometry principle, the vector projection of the closed mechanism to coordinate axis $x_{4}$ is zero. Then the following equation is satisfied.

$$
\begin{gathered}
h_{1} \cos \left(x_{1}, x_{4}\right)-s_{1} \cos \left(z_{1}, x_{4}\right)-s_{2} \cos \left(z_{2}, x_{4}\right)+h_{4}- \\
-h_{0} \cos \left(x_{0}, x_{4}\right)+s_{0} \cos \left(z_{0}, x_{4}\right)=0,
\end{gathered}
$$

in which,

$$
\left\{\begin{array}{l}
\cos \left(x_{1}, x_{4}\right)=[1,0,0]\left[C_{12}\right]\left[C_{23}\right]\left[C_{34}\right][1,0,0]^{T} \\
\cos \left(z_{1}, x_{4}\right)=[1,0,0]\left[C_{12}\right]\left[C_{23}\right]\left[C_{34}\right][0,0,1]^{T} \\
\cos \left(z_{2}, x_{4}\right)=[1,0,0]\left[C_{23}\right]\left[C_{34}\right][0,0,1]^{T} \\
\cos \left(x_{0}, x_{4}\right)=[1,0,0]\left[C_{01}\right]\left[C_{12}\right]\left[C_{23}\right]\left[C_{34}\right][1,0,0]^{T} \\
\cos \left(z_{0}, x_{4}\right)=[1,0,0]\left[C_{01}\right]\left[C_{12}\right]\left[C_{23}\right]\left[C_{34}\right][0,0,1]^{T}
\end{array}\right.
$$

It is concluded that,

$$
s_{1}=s_{0}-h_{1}\left(1+\cos \alpha_{40}\right) \sin \theta_{1} / \sin \alpha_{40} .
$$

In a similar way, other motion parameters can be concluded as follows.

$$
\left\{\begin{array}{l}
\theta_{0}=\theta_{1}-180^{\circ}, \dot{\theta}_{0}=\dot{\theta}_{1} \\
\dot{\theta}_{2}=-\dot{\theta}_{1} \\
\theta_{4}=90^{\circ}-\theta_{1}, \dot{\theta}_{4}=-\dot{\theta}_{1} \\
s_{1}=s_{0}-h_{1}\left(1+\cos \alpha_{40}\right) \sin \theta_{1} / \sin \alpha_{40} \\
s_{1}=s_{4}-h_{1}\left(1+\cos \alpha_{40}\right) \sin \theta_{1} / \sin \alpha_{40} \\
\theta_{3}=180^{\circ}+\alpha_{40}
\end{array} .\right.
$$

Assuming the angular velocity of connecting shaft 2 is $-\dot{\theta}_{1}$ in relative to input shaft 1 , and the angular velocity of input shaft 1 is $\dot{\theta}_{1}$ in relative to the stander, the angular velocity of connecting shaft 2 is 0 in relative to stander, which means the connecting shaft only translates in space. In the same way, the angular velocity of connecting shaft 3 is $\dot{\theta}_{4}=-\dot{\theta}_{1}$ in relative to input shaft 4 , and the angular velocity of input shaft 4 is set as $\dot{\theta}_{0}$ (relative to the stander). Thus, relative to the stander, connecting shaft 3 only translates in space. However, the motion features of the spatial mechanism are never related to intersection angle $\alpha_{40}$. Therefore, the transmission relationship will not change, even though the intersection angle $\alpha_{40}$ changes in the process.

Consequently, translational motions of the connecting shafts " 2 " and " 3 " in space are achieved with the RCRCR mechanism. Fixing the planetary gear $\mathrm{O}_{2}$ in Fig. 1 and the connecting shaft " 3 " in Fig. 2 together, the planetary gear $\mathrm{O}_{2}$ would translate together with the connecting shaft " 3 ", instead of revolving around axis "c". Furthermore, connecting the centre gear with the stander along the axis "d", the angular velocity relationship between the center gear and the carrier is determined by Eq. (4).

As $\omega_{H}=\omega_{a}, \omega_{2}=0$, the transmission ratio in Eq. (4) is concluded below.

$$
i=\frac{\omega_{H}}{\omega_{1}}=\frac{\omega_{a}}{\omega_{1}}=\frac{z_{1}}{z_{1}-z_{2}} .
$$


From the equation above, the transmission ratio of the reducer would never vary with the intersection angle and the distance between the shafts " 1 " and " 2 " in the process of transmission. Thus, it is a universal speed reducer maintaining a stable transmission ratio, which is adaptive to different intersect angles. If intersect distance $d_{0}=d_{1}=0$, then the transmission shafts axes "a" and "d" intersect with each other; and if distance $d_{0}=d_{1} \neq 0$, then shafts axes "a" and "d" are the spatial crossing.

In conclusion, the RCRCR mechanism shown in Fig. 3 is a 1-DOF spatial mechanism adaptive to variable intersection angles and distances, achieving the required translational motion of the planetary gear in the universal reducer. In addition, the motion pairs in the mechanism are all low pairs, which is beneficial to improve wear-resisting performance.

\section{SIMULATION RESULTS AND DISCUSSION}

To prove the function of the speed reduction transmission of the mechanism, a simulation model of the mechanism is established in Automatic Dynamic Analysis of Mechanical Systems (ADAMS) software. The simulation model is depicted in Fig. 5 in detail. To show the structure inside the speed reducer clearly, the gearbox is shown as partially transparent. Taking Figs. 3 and 4 as references, the input shaft "a" is designed with a flange, while the output shaft, which is in alignment with "d", is designed fixing with the centre gear. For the convenience of manufacturing and testing, let the intersection distance $d_{0}=d_{1}=0$. The centre pair of connecting shafts corresponds to shafts "b" and "c" in Fig. 3. The offset of the input shaft "a" and the centre connecting shaft "b" is equal to the offset of the output shaft "d" and the centre connecting shaft "c", which is the rotational radius of the planetary carrier. Since the offset is so small while a large driving torque is needed, three additional connecting shafts are introduced, distributed on a larger circle equally to help the connecting shaft revolve and thus to ensure the reducer work smoothly. Furthermore, it is beneficial to improve supporting capacity and avoid the phenomenon of "lock up" mentioned above as they distribute the load more evenly. The gearbox not only performs the function of a hermetically sealed chamber but also that of a transmission component.

The necessary parameters of the simulation model are listed in Table 1.

Table 1. Universal reducer parameters

\begin{tabular}{lc}
\hline Parameters & Values \\
\hline Planetary gear teeth & 29 \\
\hline Center gear teeth & 34 \\
\hline Module of gear [mm] & 2 \\
\hline Eccentric offset [mm] & 5 \\
\hline Transmission ratio & 6.8 \\
\hline
\end{tabular}

Figs. 6 and 7 illustrate the angular velocities of the input shaft, output shaft, and planetary gear of the speed reduction mechanism with the intersect angles $\alpha=135^{\circ}$ and $\alpha=160^{\circ}$, respectively, simulating with the same parameters, including simulation period and input velocity.

Comparing the simulation results in Figs. 6 and 7 , we can prove the translational motion of the

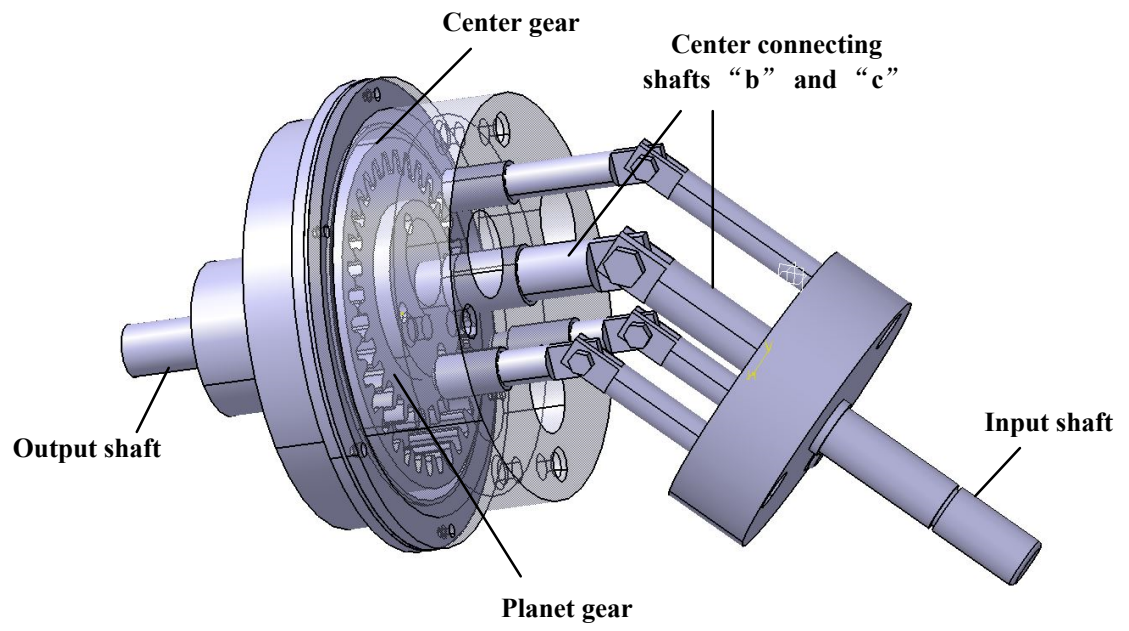

Fig. 5. Simulation model of the mechanism 


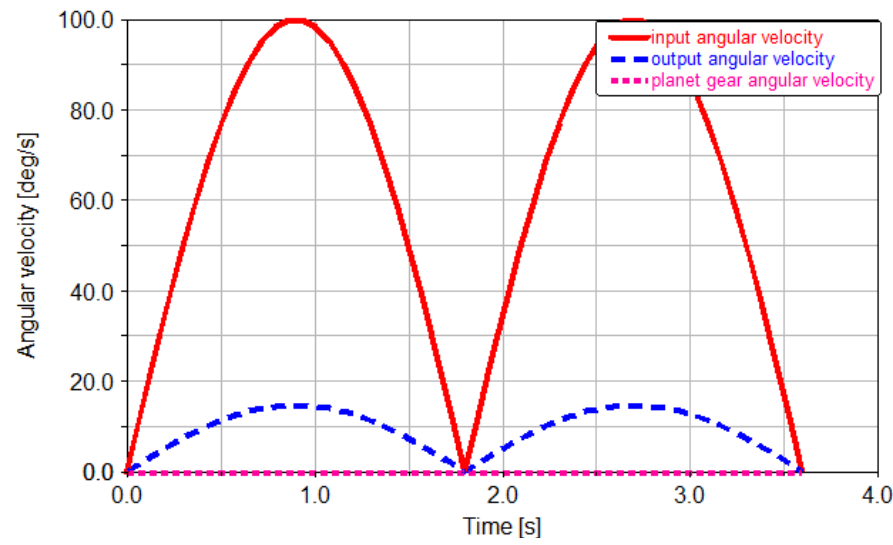

Fig. 6. Angular velocities of the input and output shafts with $\alpha=135^{\circ}$

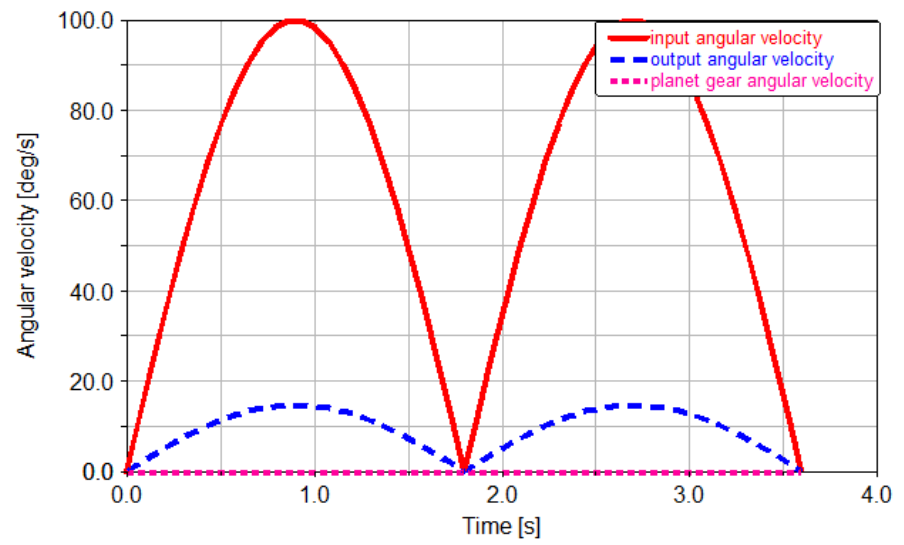

Fig. 7. Angular velocities of the input and output shafts with $\alpha=160^{\circ}$

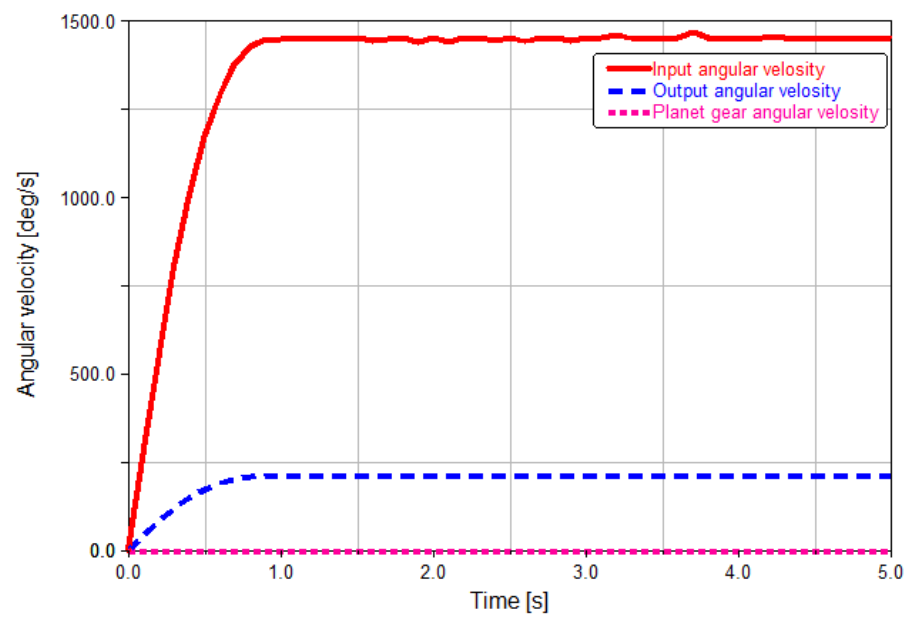

Fig. 8. Step torque response of the universal reducer

connecting shafts in the RCRCR mechanism by testing its angular velocities. It is concluded that the mechanism, which maintains the stable transmission ratio of 6.8 , can be adaptive to different intersect angles, even the intersection angle changes in the process of transmission, which means the universal reducer presents brilliant robustness with the position perturbations of input and output shafts. It is also a significant advantage in the applications of the variable intersection angle between the input and 
output shafts during transmission, which is common in transmission close to wheel for electric vehicles. To suppress the negative effects of a heavy unsprung mass in electric vehicles, a common practice is to arrange the motor on the vehicle framework as the sprung mass. Consequently, the universal reducer is introduced to transmit the power between two floating components perfectly without a constant velocity universal joint.

The torque fluctuation is also an important performance aspect for a speed reducer. A step torque response simulation is carried out, in which the initial input torque is $100 \mathrm{~N} \cdot \mathrm{m}$ and changes to zero at the time of 1 second; the whole simulation lasts 5 seconds.

From the angular velocity results shown in Fig. 8 , the output angular velocity is much smoother than the input angular velocity, while the transmission ratio remains stable. It is concluded that the universal speed reducer with the few teeth difference planetary gear has an excellent performance of torque fluctuation tolerance.

Note that the working condition of the reducer is complex, including the varying loads and misalignment of each element, especially the connecting shafts and cylinders. All the above may influence the transmission smoothness and reliability, so it is important to emphasize manufacture and assembly precision.

\section{PROTOTYPE DESIGN}

\subsection{Structure Design}

To test the speed reducer performances with the few teeth difference planetary gear mechanism, the detailed design is carried out. The parameters of the universal reducer are the same as those used in the simulation shown in Table 1. The universal reducer prototype is designed in Fig. 9, and the components are listed in Table 2. The oil-less bearing is adopted for a compact structure. The reference diameters of the centre gear and planetary gear are $68 \mathrm{~mm}$ and 58 $\mathrm{mm}$ respectively, while the distribution diameter of the three additional connecting shafts is $52 \mathrm{~mm}$. The offset of the input shaft and the centre connecting shaft is equal to the offset of the output shaft and the centre connecting shaft, which are both $5 \mathrm{~mm}$. The diameters of the input shaft and output shaft are both $25 \mathrm{~mm}$. The radius of the centre connecting shaft and the three additional connecting shafts are $15 \mathrm{~mm}$ and $10 \mathrm{~mm}$, respectively. The gearbox radius is $53 \mathrm{~mm}$, and the overall thickness is $84 \mathrm{~mm}$. In conclusion, the designed universal reducer has the advantage of a compact structure.

Table 2. Prototype components

\begin{tabular}{|c|c|c|c|}
\hline No. & Name & No. & Name \\
\hline 1 & Input shaft & 13 & Planetary gear \\
\hline 2 & Elastic collar & 14 & Reducer shell \\
\hline 3 & Deep groove ball bearing & 15 & Center gear \\
\hline 4 & Bush & 16 & Deep groove ball bearing \\
\hline 5 & Oil-less bearing & 17 & Elastic collar \\
\hline 6 & Oil-less bearing & 18 & Deep groove ball bearing \\
\hline 7 & Connecting shaft & 19 & Bush \\
\hline 8 & Connecting shaft & 20 & Bush \\
\hline 9 & Bolt & 21 & Bolt \\
\hline 10 & Connecting shaft & 22 & Rubber gasket \\
\hline 11 & Planetary carrier & 23 & Socket head cap screw \\
\hline 12 & Reducer shell & & \\
\hline
\end{tabular}

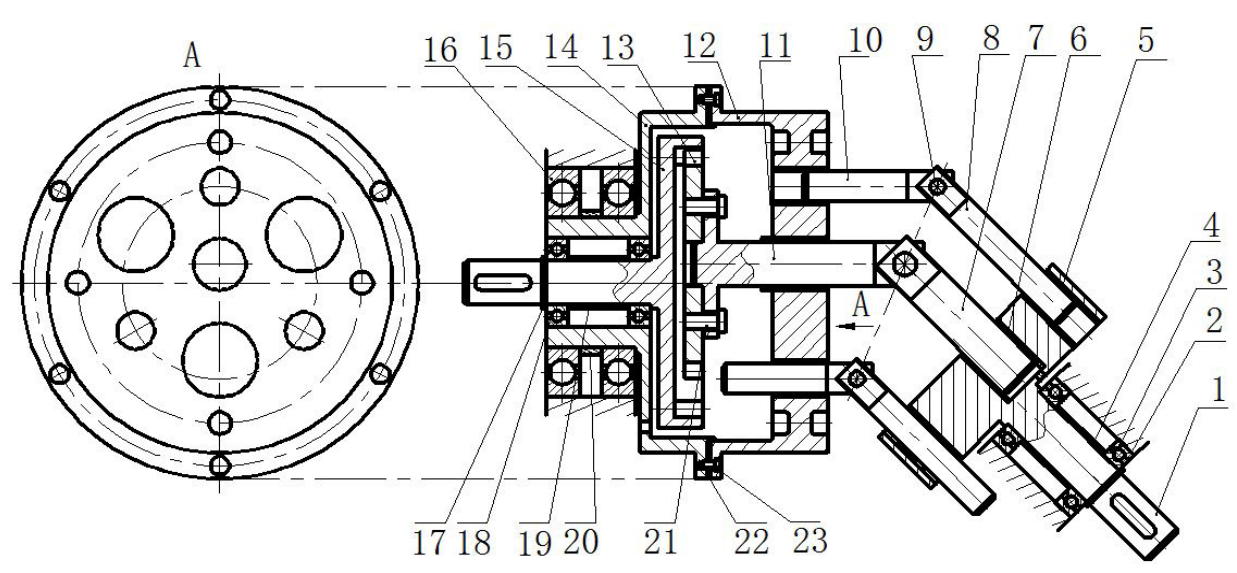

Fig. 9. Speed reducer prototype 


\subsection{Strength Analysis}

A torque $M_{\text {in }}$ is applied on the input shaft, while the load on the output shaft is $M_{\text {out }}$. With the transmission ratio $i$, Eq. (18) is concluded.

$$
M_{\text {out }}=i \cdot M_{\text {in }} .
$$

Firstly, taking the connecting shafts (marked as components 2 and 3) as a whole object, the forces and torques applied to the object are depicted in Fig. 2. The distance between $\mathrm{B}$ and $\mathrm{E}$ is $L_{1}$, the same as the distance between $\mathrm{C}$ and $\mathrm{E}$. The angle between the connecting shafts 2 and 3 is $2 \alpha$, and the distance between the input shaft and the connecting shaft is $r$, which equals to the distance between the output shaft and the connecting shaft (component 3 ).

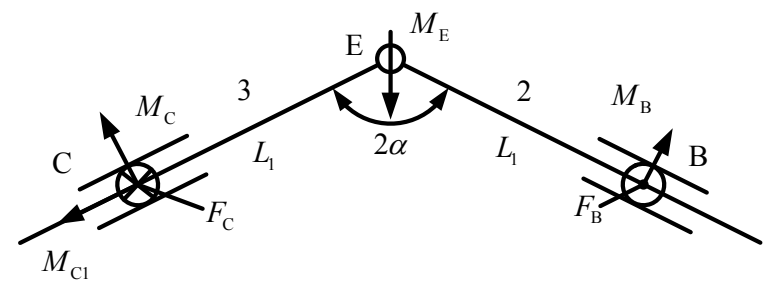

Fig. 10. Dynamic analysis of component 2 and 3

In the rotating process, when the connecting shafts are located at the highest position, with the involvements of $F_{\mathrm{B}}$ and $F_{\mathrm{C}}$ on the shafts 2 and 3, to keep the translational motion of the connecting shafts, a torque of $M_{\mathrm{E}}$ is generated on point $\mathrm{E}$.

$$
M_{\mathrm{E}}=2 F_{\mathrm{B}} L \sin \alpha,
$$

in which, $F_{\mathrm{B}}=M_{i n} / r$.

A torque of $M_{\mathrm{C} 1}$ is applied on point $\mathrm{C}$ under the tangential force of the planetary gear.

$$
\left\{\begin{array}{l}
M_{\mathrm{C} 1}=F_{t} r_{2} \\
F_{t}=2 M_{\text {out }} /\left(m z_{1}\right)
\end{array}\right.
$$

in which, $F_{t}$ is the tangential force, $m$ is the module of the internal gear and $z_{1}$ is the teeth of the internal gear.

According to the kinematic analysis, to guarantee the translational motion of the connecting shafts, the torques should be kept balanced as follows.

$$
\left\{\begin{array}{l}
M_{\mathrm{B}} \sin \alpha+M_{\mathrm{C}} \sin \alpha-M_{\mathrm{Cl}} \cos \alpha-M_{\mathrm{E}}=0 \\
M_{\mathrm{B}} \cos \alpha-M_{\mathrm{C}} \cos \alpha-M_{\mathrm{Cl}} \sin \alpha=0
\end{array} .\right.
$$

The torques $M_{\mathrm{B}}$ and $M_{\mathrm{C}}$ can be concluded according to the equations above.

Clearly, the critical position of connecting shaft 3 locates $\mathrm{C}$ point. The connecting shaft (component 3 in Fig. 10) is torqued by $M_{C 1}$ and bent by $M_{\mathrm{C}}$ simultaneously. $45 \#$ steel with the allowable bending stress of $60 \mathrm{MPa}$ is preferred. Based on the third strength theory, the equation below should be satisfied.

$$
\sigma_{c a}=\sqrt{\left(\frac{M}{W}\right)^{2}+4\left(\frac{a T}{2 W}\right)^{2}}=\frac{\sqrt{M_{\mathrm{C}}^{2}+\left(0.6 M_{\mathrm{Cl}}\right)^{2}}}{0.1 d^{3}},
$$

in which, $W$ is the section coefficient in bending. Considering the torsional shear stress is the pulsating cycle stress, $a$ is determined as 0.6.

As the prototype is designed in a much smaller size than the real universal reducer, the output torque is about $34 \mathrm{~N} \cdot \mathrm{m}$. Given $\alpha=60^{\circ}, d=15 \mathrm{~mm}$, the bending strength:

$$
\sigma_{c a 3}=52.4 \mathrm{MPa} .
$$

For the component 2 in Fig. 10, it is only bent by $M_{\mathrm{B}}$. Consequently, the bending strength is computed as follows, while the allowable bending fatigue stress is $275 \mathrm{MPa}$.

$$
\sigma_{c a 2}=\frac{M_{\mathrm{B}}}{0.1 d^{3}}=158.5 \mathrm{MPa} .
$$

Similarly, the strength analyses of other main components can be carried out through force analyses. The emphasis and contribution of this paper are to propose a novel mechanism with the function of universal reduction. Based on the planetary gear and RCRCR spatial mechanism, a prototype with a smaller ratio is designed. Consequently, the results of other main components are listed in Table 3.

Table 3. Main components strength analyses

\begin{tabular}{lcc}
\hline Components & $\begin{array}{c}\text { Allowable strength } \\
{[\mathrm{MPa}]}\end{array}$ & $\begin{array}{c}\text { Actual strength } \\
{[\mathrm{MPa}]}\end{array}$ \\
\hline Input shaft 1 & 60 & 47.04 \\
\hline Connecting shaft 7 & 275 & 158.52 \\
\hline Connecting shaft 10 & 60 & 52.45 \\
\hline Center gear 15 & 60 & 45.35 \\
\hline
\end{tabular}

For the strength of the planetary and internal gears, the equations below are satisfied respectively [17].

$$
\begin{gathered}
\sigma_{F 1}=\frac{2 K K_{p} T_{g} Y_{F 1}}{b d_{g} m} \leq \sigma_{F P 1}, \\
\sigma_{F 2}=\frac{Y_{F 2}}{Y_{F 1}} \sigma_{F 1} \leq \sigma_{F P 2},
\end{gathered}
$$

in which, $K$ is the load efficiency, $K_{p}$ is the uneven load distribution coefficient, $T_{g}$ is the torque of the planetary gear, $Y_{\mathrm{F} 1}$ and $Y_{\mathrm{F} 2}$ are tooth shape coefficients 
of planetary and internal gears respectively, $b$ is the tooth width, $d_{g}$ is the pitch circle diameter of the planetary gear, and $m$ is the gear module.

According to the parameters of the gears and the torque, the bending strengths of the planetary and internal gears are 213.84 MPa and 201.6 MPa, while the allowable strengths are $238 \mathrm{MPa}$ and $303 \mathrm{MPa}$ respectively.

According to the strength analysis results of the main components above, it is concluded that the strength requirement of the universal reducer prototype is satisfied.

\subsection{Manufacturing and Lubrication}

For the manufacturing of the proposed universal reducer, the importance of the RCRCR spatial mechanism manufacturing should be especially emphasized as it plays an important role. In theory, the connecting points of the transmission shafts should be strictly located in the same plane, as depicted by the dotted line depicted in Fig. 9. In addition, the poor assembly between the shaft and hole may contribute to the failure of the reducer. Combining the two aspects above, in the RCRCR mechanism, more precision is required between the connecting relationship between 8 and 10 than that of 8 and 7. Consequently, for the components on both sides of the plane, to guarantee equal manufacturing precision and assembly tolerance based on the same standard, the holes corresponding to the connecting shafts can be manufactured simultaneously, as depicted in Fig. 11.

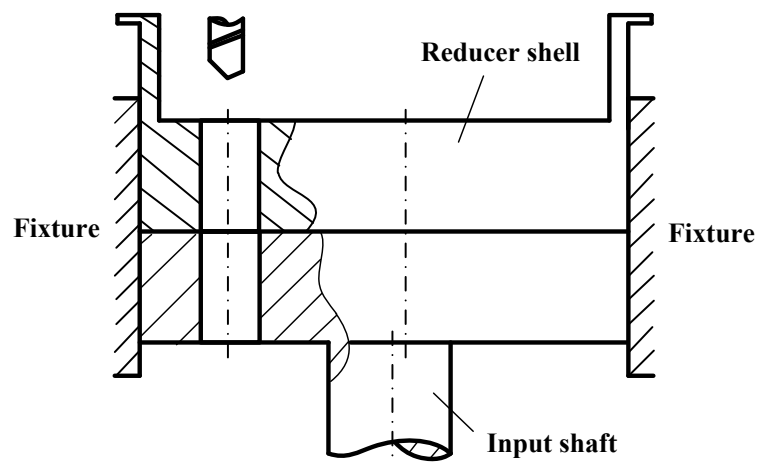

Fig. 11. Simultaneous manufacturing

The assembly tolerance can be $\mathrm{H} 7 / \mathrm{h} 7$. Furthermore, feasible manufacturing processing to improve the manufacturing precision should be involved, such as grinding and polishing.

Lubrication is beneficial for the reductions of friction and temperature. Consequently, system efficiency and working life are improved. In addition to low load capacity in common working conditions, the low circumferential speed also contributes to the application characteristics. Combining the two factors above, grease is preferred in the lubrication of planetary gear reducer and deep groove ball bearing, while no lubrication is needed for the oil-less bearings.

\section{CONCLUSIONS}

A novel speed reduction mechanism with selfadaptability to variable intersection angles is proposed in this paper, which is integrated by the few teeth difference planetary gear mechanism and the RCRCR spatial mechanism, achieving the translational motion of the planetary gear. Another three pairs of the connecting shafts are added to the mechanism to improve the supporting capacity and the homogeneity of load distribution. Through theoretical analysis and simulations, it is proved that the speed reduction mechanism proposed maintains a stable transmission ratio; even the intersection angle varies in the process. The detailed design is conducted for manufacture. For the transmission smoothness and reliability, the precision of manufacture and assembly is of much importance. It has significantly wide applications of real-time speed reduction in poor working environments for transmission shafts.

However, some work remains to be completed and improved. The mechanical efficiency of the universal reducer should be tested. Furthermore, for better application and spread, lightweight design and strength analysis need to be carried out to improve structure compactness and integration.

\section{ACKNOWLEDGMENTS}

The authors gratefully acknowledge the support by the National Natural Science Foundation of China under Grant No. 51375344.

\section{REFERENCES}

[1] Wu, Y.C., Chen, L.A. (2015) Design of an 8-speed internal gear hub with a rotary control mechanism for bicycles. Tehnički vjesnik - Technical gazette, vol. 22, no. 4, p. 865-871, DOI:10.17559/TV-20131225075135.

[2] Blagojevic, M, Marjanovic, N, Djordjevic, Z, Stojanovic, B., Disic, A. (2011). A new design of a two-stage cycloidal speed reducer. Journal of Mechanical Design, vol. 133, no. 8, p. 085001, D0I:10.1115/1.4004540.

[3] Blagojevic, M., Kocic, M., Marjanovic, N, Stojanovic, B., Dordevic, Z., Ivanovic, L., Marjanovic, V. (2012). Influence of the friction on the cycloidal speed reducer efficiency. Journal 
of the Balkan Tribological Association, vol. 18, no. 2, p. 217 227.

[4] Concli, F., Gorla, C. (2013). Influence of lubricant temperature, lubricant level and rotational speed on the churning power loss in an industrial planetary speed reducer: Computational and experimental study. International Journal of Computational Methods and Experimental Measurements, vol. 1, no. 4, p. 353-366, DOI:10.2495/CMEM-V1-N4-353-366.

[5] Wang, J., Kong, L., Liu, B., Hu, X., Yu, X. Kong, W. (2014). The mathematical model of spiral bevel gears - A review. Strojniški vestnik - Journal of Mechanical Engineering, vol. 60, no. 2, p. 93-105, D0l:10.5545/sv-jme.2013.1357.

[6] Zhang, Y., Yan, H., Zeng, T. (2015). Computerised design and simulation of meshing and contact of formate hypoid gears generated with a duplex helical method. Strojniški vestnik Journal of Mechanical Engineering, vol. 61, no. 9, p. 523-532, DOI:10.5545/sv-jme.2015.2627.

[7] Dudás, L. (2013). Modelling and simulation of a new worm gear drive having point-like contact. Engineering with Computers, vol. 29, no. 3, p. 251-272, D0l:10.1007/s00366012-0271-0.

[8] Ding, J., Chen, Y., Lv, Y., Song, C. (2014). Position-parameter selection criterion for a helix-curve meshing-wheel mechanism based on sliding rates. Strojniški vestnik - Journal of Mechanical Engineering, vol. 60, no. 9, p. 561-570, D0I:10.5545/sv-jme.2013.1574.

[9] Chen, X.B., Li, Y. (2004). Speed Up/Down Mechanism of Universal Transmission Based on Internal Planetary Gear. Patent No. 200410024618.8, State Intellectual Property Office of the P.R.C., Beijing.
[10] Litvin, F.L., Tan, J. (1989). Singularities in motion and displacement functions of constrained mechanical systems. The International Journal of Robotics Research, vol. 8, no. 2, p. 30-43, DOl:10.1177/027836498900800203.

[11] Chen, X.B., Li, Y. (2004). Speed Up/Down Mechanism of Universal Transmission Based on Planetary Synchronous Pulley. Patent No. 200410024617.3, State Intellectual Property Office of the P.R.C., Beijing.

[12] Chen, X.B., Wang, B., Chen, M.F., Li, Y. (2012). An innovative speed reduction mechanism with self-adaptability to variable transmission angles. Mechanism and Machine Theory, vol. 48, p. 41-51, D0I:10.1016/j.mechmachtheory.2011.09.004.

[13] Chen, X.B., Li, Y. (2004). Speed Up/Down Mechanism of Universal Transmission Based on External Planetary Gear, Patent No. 200410024619.2, State Intellectual Property Office of the P.R.C., Beijing.

[14] Li, S. (2008). Contact problem and numeric method of a planetary drive with small teeth number difference. Mechanism and Machine Theory, vol. 43, no. 9, p. 10651086, D0l:10.1016/j.mechmachtheory.2007.10.003.

[15] Chen, E., Walton, D. (1990). The optimum design of KHV planetary gears with small tooth differences. International Journal of Machine Tools and Manufacture, vol. 30, no. 1, p. 99-109, DOI:10.1016/0890-6955(90)90045-K.

[16] Xiao-Long, S. (1995). Determination of load sharing factor for planetary gearing with small tooth number difference. Mechanism and Machine Theory, vol. 30, no. 2, p. 313-321, DOI:10.1016/0094-114X(93)E0007-S.

[17] Pu, L.G. (1994). Mechanical Design. Higher Education Press, Beijing. 